Cite this: Polym. Chem., 2014, 5, 4061

\title{
Functional 2-methylene-1,3-dioxepane terpolymer: a versatile platform to construct biodegradable polymeric prodrugs for intracellular drug delivery $\dagger$
}

\author{
Tongjiang Cai, ${ }^{a}$ Yangjun Chen, ${ }^{a}$ Yin Wang, ${ }^{a}$ Haibo Wang, ${ }^{a}$ Xiangsheng Liu, ${ }^{a}$ Qiao Jin, ${ }^{* a}$ \\ Seema Agarwal ${ }^{\mathrm{b}}$ and Jian $\mathrm{Ji}^{\star a}$
}

Functional 2-methylene-1,3-dioxepane (MDO) terpolymers were explored here as a versatile platform to construct biodegradable $\mathrm{pH}$ sensitive polymeric prodrugs for intracellular drug delivery. A series of MDO-based biodegradable functional polyester P(MDO-CO-PEGMA-CO-PDSMA) with different compositions were synthesized by terpolymerization of MDO, poly(ethylene glycol) methyl ether methacrylate (PEGMA) and pyridyldisulfide ethylmethacrylate (PDSMA) via a simple one-pot radical ringopening copolymerization. Mal-DOX, which contains a $\mathrm{pH}$-sensitive hydrazone bond between doxorubicin (DOX) and the maleimide group, was covalently conjugated in one pot to free thiol groups of PDSMA units via thiol-ene click chemistry in the presence of tri(2-carboxyethyl)phosphine (TCEP). The DOX-conjugated P(MDO-CO-PEGMA-CO-PDSMA) can self-assemble into prodrug micelles. The diameter and morphology of the polymeric prodrug micelles were measured by dynamic light scattering (DLS) and transmission electron microscopy (TEM). Because of the existence of the $\mathrm{pH}$-sensitive hydrazone bonds, in vitro drug release results showed that the release of DOX was much faster at $\mathrm{pH} 5.5$ than that at $\mathrm{pH}$ 7.4. Flow cytometry and fluorescence microscopy demonstrated that the prodrug micelles could be efficiently internalized by cancer cells. In vitro cytotoxicity showed that the DOXconjugated prodrug micelles can strongly inhibit the proliferation of cancer cells remarkably. Importantly, this work provides a versatile strategy for the fabrication of biodegradable polymeric prodrug nanocarriers.

Received 20th February 2014 Accepted 21st March 2014

DOI: $10.1039 / \mathrm{c} 4 \mathrm{py} 00259 \mathrm{~h}$

www.rsc.org/polymers

\section{Introduction}

Biodegradable polymers have been widely used in biomedical fields, such as tissue engineering, biomedical implants, and drug delivery. ${ }^{1-4}$ Among the biodegradable polymers, aliphatic polyesters are well studied and frequently used in biomedical areas. $^{5}$ Poly( $\varepsilon$-caprolactone) (PCL), polylactide (PLA), polyglycolide (PGA), and poly(lactide-co-glycolide) (PLGA) are the most well-known biodegradable polyesters that have been approved by the US Food and Drug Administration (FDA). However, the lack of functional groups in those aliphatic polyesters has limited their potential in the design of new functional polymer with good biocompatibility and biofunctionality. Radical ring-opening polymerizations (RROPs) of cyclic ketene

${ }^{a}$ MOE Key Laboratory of Macromolecule Synthesis and Functionalization of Ministry of Education, Department of Polymer Science and Engineering, Zhejiang University, Hangzhou, 310027, China.E-mail:jinqiao@zju.edu.cn; jijian@zju.edu.cn

${ }^{b}$ University of Bayreuth, Macromolecular Chemistry II, Bayreuth Center for Colloids and Interfaces, Universitätsstrasse 30, 95440, Bayreuth, Germany

$\dagger$ Electronic supplementary information (ESI) available: The detailed synthesis and characterizations of MDO, PDSMA, and Mal-DOX. ${ }^{13} \mathrm{C}$ NMR of P3. See DOI: 10.1039/c4py00259h acetals, such as 2-methylene-1,3-dioxepane (MDO), have recently attracted significant interest for preparation of biodegradable aliphatic polyesters. ${ }^{6-14}$ This polymerization reaction provides the new possibility to combine ester units with the $\mathrm{C}-\mathrm{C}$ backbone of vinyl polymers in a random way to generate a new class of biodegradable materials [poly(vinyl-co-ester)s]. Most importantly, functional polyesters can be facilely synthesized just by copolymerization of cyclic ketene acetals with functional vinyl polymers. In our previous research, photo cross-linkable coumarin groups were introduced to the MDO copolymers and photo cross-linked biodegradable micelles can be easily prepared. ${ }^{15}$ Very recently, functionalized polyesters poly(MDOco-GMA) were achieved by copolymerization of MDO and glycidyl methacrylate (GMA). The epoxy functionalized poly(MDOco-GMA) can undergo a number of chemical reactions. Different molecules, such as heparin, can be covalently immobilized to poly(MDO-co-GMA) copolymers. ${ }^{16}$

Polymeric prodrug micelles have received extensive attention as drug nanocarriers. ${ }^{17-23}$ Since the drug is covalently conjugated to the polymer, polymeric prodrugs are more stable than traditional physically encapsulated drugs during circulation. For the purposes of drug delivery vehicles, it is imperative for 
prodrug micelles to have good biodegradability properties because the low-molecular weight degradation products are expected to be metabolized or excreted, and not to cause longterm toxicity. In recent years, biodegradable polymers have been used to fabricate polymeric prodrug delivery systems. ${ }^{24}$ Wang and co-workers reported a doxorubicin (DOX) prodrug with a biodegradable polyphosphoester as the back bone. ${ }^{25}$ The dual pH-sensitive polymeric prodrug micelles exhibited efficient anticancer drug delivery. Our group designed a phosphorylcholine biomimic $\mathrm{pH}$-sensitive polymeric prodrug based on biodegradable polycarbonate for efficient intracellular drug delivery. ${ }^{26}$ However, polyester-based polymeric prodrug micelles are seldom reported. In this research, we constructed biocompatible amphiphilic functional polyesters that can be used to covalently conjugate DOX. The functional polyester, $\mathrm{P}\left(\mathrm{MDO}-\mathrm{co}^{-}\right.$ PEGMA-co-PDSMA), was synthesized via a simple one-pot radical ring-opening terpolymerization of 2-methylene-1,3dioxepane (MDO), poly(ethylene glycol) methyl ether methacrylate (PEGMA) and pyridyldisulfide ethylmethacrylate (PDSMA). Mal-DOX, which contains a $\mathrm{pH}$-sensitive hydrazone bond between the drug and the maleimide group, was covalently conjugated in one pot to free thiol groups of PDSMA units in the presence of a disulfide reducing agent (Scheme 1). The biodegradable, biocompatible, and $\mathrm{pH}$-sensitive polymeric prodrug micelles were prepared by the self-assembly of amphiphilic DOX-conjugated P(MDO-co-PEGMA-co-PDSMA). The novel polyester-based polymeric prodrug micelles were successfully applied in the delivery of DOX. We hypothesize here that the facile introduction of functional polyester into polymeric prodrug systems will provide a novel approach to develop more sophisticated drug nanocarriers that might have great potential in biomedical applications.

\section{Experimental section}

\section{Materials}

Poly(ethylene glycol) methyl ether methacrylate (PEGMA, $M_{\mathrm{n}} \sim$ 475, Sigma-Aldrich) was passed through a basic alumina to remove the initiator. Doxorubicin hydrochloride $(\mathrm{DOX} \cdot \mathrm{HCl})$

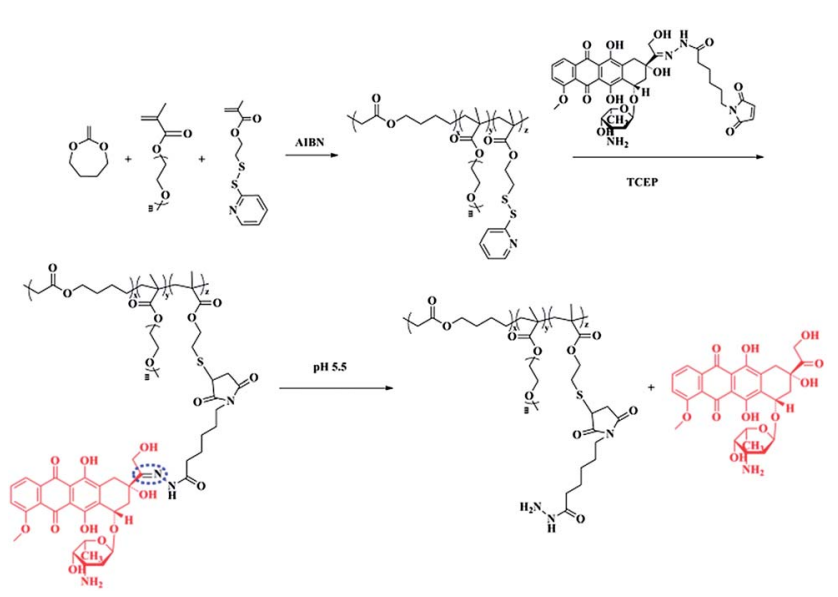

Scheme 1 Schematic illustration of the synthesis of DOX-conjugated P(MDO-CO-PEGMA-CO-PDSMA). was purchased from Zhejiang Hisun Pharmaceutical Co., Ltd. 2-Methylene-1,3-dioxepane (MDO) was synthesized according to the published procedure. ${ }^{27}$ Azobis(isobutyronitrile) (AIBN, Sigma-Aldrich) was recrystallized from methanol prior to use. Neutralized tri(2-carboxyethyl)phosphine (TCEP) was prepared according to the literature. ${ }^{28}$ Maleimide derivative of doxorubicin (Mal-DOX) that contains a hydrazone bond between the drug and the maleimide group was synthesized according to the procedure reported previously. ${ }^{28,29}$ Pyridyldisulfide ethylmethacrylate (PDSMA) was synthesized as described previously. ${ }^{30}$ All other reagents were obtained from commercial suppliers and used without further purification.

\section{Synthesis of P(MDO-co-PEGMA-co-PDSMA)}

In a typical reaction, the radical ring-opening terpolymerization of MDO, PEGMA and PDSMA was carried out in a $50 \mathrm{~mL}$ Schlenk tube at a temperature of $70{ }^{\circ} \mathrm{C}$ using AIBN (1 wt\%) as the initiator. PEGMA (0.3 g, $0.63 \mathrm{mmol}$ ), PDSMA (0.322 g, 1.26 $\mathrm{mmol}$ ), MDO (0.72 g, $6.32 \mathrm{mmol}$ ), AIBN (13.42 $\mathrm{mg}$ ) and $2 \mathrm{~mL}$ toluene was added to a $50 \mathrm{~mL}$ flask. The solution was degassed by three freeze-pump-thaw cycles and then placed in a preheated oil bath at $70{ }^{\circ} \mathrm{C}$. After $12 \mathrm{~h}$, the reaction was stopped by transferring the Schlenk tube to liquid nitrogen. The polymer was purified by precipitation twice into ether and then dried under vacuum at $40{ }^{\circ} \mathrm{C}$.

\section{Synthesis of DOX-conjugated P(MDO-co-PEGMA-co-PDSMA)}

In order to conjugate DOX to the terpolymer, $100 \mathrm{mg} \mathrm{P}\left(\mathrm{MDO}-\mathrm{co}^{-}\right.$ PEGMA-co-PDSMA) and $24 \mathrm{mg}$ Mal-DOX were dissolved in $3 \mathrm{~mL}$ dried chloroform and $1 \mathrm{~mL}$ dried methanol in a $50 \mathrm{~mL}$ roundbottom flask. Then, $8 \mathrm{mg}$ neutralized TCEP in $2 \mathrm{~mL}$ dried methanol was added dropwise into the flask. After stirring overnight, the reaction solution was dialyzed against methanol for $24 \mathrm{~h}$ and deionized water for another $24 \mathrm{~h}$.

\section{Enzymatic degradation of terpolymer P(MDO-co-PEGMA-co- PDSMA)}

$\mathrm{P}$ (MDO-co-PEGMA-co-PDSMA) (5 mg) was added to $10 \mathrm{~mL}$ PBS $(0.1 \mathrm{M}, \mathrm{pH}=7.4)$, and lipase from porcine liver was then added to a final concentration of $2 \mathrm{mg} \mathrm{mL}^{-1}$. This mixture was then placed at $37^{\circ} \mathrm{C}$ with gentle shaking for 3 days. The solution was lyophilized and then dissolved in $10 \mathrm{~mL}$ THF. After filtering with a $0.45 \mu \mathrm{m}$ Millipore filter, the solution was used to measure the molecular weight by GPC measurement.

\section{Preparation of polymeric micelles}

DOX-conjugated P(MDO-co-PEGMA-co-PDSMA) (15 mg) was first dissolved in $2 \mathrm{~mL}$ THF. After stirring for $0.5 \mathrm{~h}, 2 \mathrm{~mL}$ distilled water was added dropwise and stirred for another $3 \mathrm{~h}$. The solution was then dialyzed against distilled water for 2 days before use.

\section{In vitro $\mathrm{pH}-$ responsive drug release}

The in vitro release of DOX from prodrug micelles was conducted under different $\mathrm{pH}$ media at $37{ }^{\circ} \mathrm{C}$. In a typical experiment, $2 \mathrm{~mL}$ of prodrug micellar solution $\left(1 \mathrm{mg} \mathrm{mL}^{-1}\right)$ was added 
into a dialysis bag $(\mathrm{MWCO}=3500)$. The dialysis bag was then immersed into $10 \mathrm{~mL}$ PBS at $\mathrm{pH} 5.0$ or $\mathrm{pH} 7.4$ with constant shaking $(100 \mathrm{rpm})$ at $37{ }^{\circ} \mathrm{C}$. At fixed time intervals, $0.5 \mathrm{~mL}$ released media was taken out and replaced with fresh PBS medium. The DOX concentrations in samples were then measured by fluorometric measurement.

\section{Cell viability assays}

The biocompatibility of P(MDO-co-PEGMA-co-PDSMA) was tested by the standard 3-(4,5-dimethylthiazol-2-yl)-2,5-diphenyltetrazolium bromide (MTT) assays using human umbilical vein endothelial cells (HUVECs). HUVECs were seeded into 96-well plates (5000 cells per well) using $180 \mu \mathrm{L}$ RPMI 1640 culture media. After incubation for $48 \mathrm{~h}\left(37^{\circ} \mathrm{C}, 10 \%\right.$ humidity, $\left.5 \% \mathrm{CO}_{2}\right), \mathrm{P}(\mathrm{MDO}-\mathrm{co}$-PEGMA-co-PDSMA $)$ in PBS solution $(20 \mu \mathrm{L}$ per well) was added to form a concentration gradient of 50 to $1000 \mu \mathrm{g} \mathrm{mL} \mathrm{m}^{-1}$. For another $48 \mathrm{~h}, 20 \mu \mathrm{L}$ MTT was added into every well and incubated for $4 \mathrm{~h}$. The solution in every well was then replaced with $150 \mu \mathrm{L}$ DMSO, and the absorption values were measured using a microplate reader at $490 \mathrm{~nm}$. Data are expressed as average $\pm \mathrm{SD}(n=4)$.

To evaluate the cell cytotoxicity of DOX-conjugated $\mathrm{P}(\mathrm{MDO}-$ co-PEGMA-co-PDSMA), the prodrug micelles were used for MTT assays. Human Lung Adenocarcinoma A549 cells were seeded into 96-well plates (5000 cells per well) using F-12K $(180 \mu \mathrm{L}$ per well) as the culture medium. F-12K was supplemented with $10 \%$ fetal bovine serum. After A549 cells were incubated for $24 \mathrm{~h}$ at $37{ }^{\circ} \mathrm{C}$ in a humidified atmosphere containing $5 \% \mathrm{CO}_{2}, 20 \mu \mathrm{L}$ prodrug micellar solution was added into every well with a final DOX concentration ranging from 0.25 to $10 \mu \mathrm{g} \mathrm{mL} L^{-1}$. After incubation for another $48 \mathrm{~h}, 20 \mu \mathrm{L}$ MTT was added and incubated for another $4 \mathrm{~h}$. The culture media in each well was replaced by $150 \mu \mathrm{L}$ DMSO. The absorbance was measured at a wavelength of $490 \mathrm{~nm}$. Data are expressed as average $\pm \mathrm{SD}$ $(n=4)$. As a control, the cytotoxicity of free DOX with different concentrations was also measured by the same method.

\section{Cellular uptake}

Cellular internalization of the prodrug micelles was characterized by fluorescence microscopy and flow cytometry.

\section{Fluorescence microscopy}

A549 cells were seeded in confocal dishes $\left(2 \times 10^{4}\right.$ cells per well $)$ with $1 \mathrm{~mL} \mathrm{F-12K}$ culture media and incubated for $24 \mathrm{~h}$. The prodrug micelles and free DOX were then added at an equivalent DOX concentration of $10 \mu \mathrm{g} \mathrm{mL}{ }^{-1}$. The cells were incubated for $2 \mathrm{~h}, 4 \mathrm{~h}$, and $6 \mathrm{~h}$. The medium was removed, and the confocal dishes were washed three times with fresh PBS. The cells were fixed with $4 \%$ formaldehyde for $20 \mathrm{~min}$, and the cell nuclei were dyed with 4,6diamidino-2-phenylindole (DAPI) for $30 \mathrm{~min}$. The fluorescence images were observed using a fluorescence microscope.

\section{Flow cytometry}

A549 cells were incubated in 24 -well plates $\left(1 \times 10^{5}\right.$ cells per well) in F-12K media for $24 \mathrm{~h}$. The prodrug micelles and free
DOX were added with a final DOX concentration of $10 \mu \mathrm{g} \mathrm{mL}{ }^{-1}$. After incubation for $2 \mathrm{~h}, 4 \mathrm{~h}$, and $6 \mathrm{~h}$, the cells were washed three times with fresh PBS solution and treated with Trypsin. The mixture solution was centrifuged at $1000 \mathrm{rpm}$ for $5 \mathrm{~min}$ to remove the solution. The cells were resuspended in $0.5 \mathrm{~mL}$ PBS for the next detection. Samples of $1 \times 10^{4}$ cells were collected using a FACSCalibur flow cytometer. The results were analyzed using WinMDI 2.9 software.

\section{Characterization}

${ }^{1} \mathrm{H}$ NMR spectra were recorded on a Bruker DMX500 instrument. Molecular weights and molecular weight distributions of the terpolymers were measured with a gel permeation chromatography (GPC) setup comprising PL gel 10 mm MIXED-B columns using a refractive index (RI) detector and a series of narrow polystyrene standards for the calibration curve. The eluent was HLPC-grade THF, and the flow rate was $1.0 \mathrm{~mL}$ $\min ^{-1}$ at $40{ }^{\circ} \mathrm{C}$. Dynamic light scattering (DLS) measurements were performed with Zetasizer Nano-ZS from Malvern Instruments equipped with a He-Ne laser at a wavelength of $633 \mathrm{~nm}$ at $25{ }^{\circ} \mathrm{C}$. Intensity-average hydrodynamic diameter $\left(D_{\mathrm{h}}\right)$ was used in this research. Fluorescence spectra were recorded using a Perkin-Elmer LS 55 fluorescence spectrometer. Excitation and emission were set at 488 and $560 \mathrm{~nm}$, respectively, with a band width of $10 \mathrm{~nm}$. Transmission electron microscopy (TEM) measurements were performed on a JEM-3010 instrument operating at $80 \mathrm{kV}$ in bright field mode. The TEM samples were prepared by drying a drop of a dilute micellar solution onto a carbon-coated copper grid.

\section{Results and discussion}

\section{Synthesis of DOX-conjugated P(MDO-co-PEGMA-co-PDSMA)}

The biodegradable functional polyester $\mathrm{P}$ (MDO-co-PEGMA-coPDSMA) was first prepared by radical ring-opening terpolymerization of MDO, PEGMA and PDSMA using AIBN as initiator as illustrated in Scheme 1. The anticancer drug DOX was then conjugated to the functional polyester $\mathrm{P}$ (MDO-co-PEGMA-coPDSMA) via thiol-ene click chemistry in the presence of the disulfide reducing agent TCEP. A series of the terpolymers $\mathrm{P}$ (MDO-co-PEGMA-co-PDSMA) were prepared by adjusting the molar ratio of the monomers in the initial feed. The results are shown in Table 1 . The typical ${ }^{1} \mathrm{H}$ NMR results of the terpolymer $\mathrm{P} 3$ are presented in Fig. 1. As expected, well-defined $\mathrm{P}\left(\mathrm{MDO}-\mathrm{co}^{-}\right.$ PEGMA-co-PDSMA) terpolymers were obtained. From the ${ }^{1} \mathrm{H}$ NMR results in Fig. 1, the characteristic signals of MDO, PEGMA and PDSMA units can be clearly observed and all peaks can be well assigned with its chemical structures. By comparing the well-defined peak integrals of $-\mathrm{COO}-\mathrm{CH}_{2}-(3.97 \mathrm{ppm})$ of $\mathrm{MDO}$ units, $-\mathrm{OCH}_{2} \mathrm{CH}_{2}-(3.63 \mathrm{ppm})$ of PEGMA units, and $-\mathrm{C}_{5} \mathrm{H}_{4} \mathrm{~N}$ of PDSMA (7.12, 7.68, $8.47 \mathrm{ppm})$, the composition of $\mathrm{P}(\mathrm{MDO}-\mathrm{co}$ PEGMA-co-CMA) can be calculated, and the results are shown in Table 1. It was observed that the mole fraction of MDO was less in terpolymers as compared to that in the initial feed. The comparatively low reactivity ratio of MDO could be attributed to the lower stability of the corresponding MDO radical as 
Table 1 Characterization of the terpolymer P(MDO-Co-PEGMA-CO-PDSMA) with different compositions

\begin{tabular}{lllll}
\hline P(MDO-co-PEGMA-co-PDSMA) & MDO : PEGMA : PDSMA in feed (mole) & $M_{\mathrm{n}}(\mathrm{GPC})$ & $M_{\mathrm{w}} / M_{\mathrm{n}}(\mathrm{GPC})$ & MDO : PEGMA : PDSMA ( ${ }^{1} \mathrm{H}$ NMR) \\
\hline P1 & $10: 1: 1$ & 11500 & 2.32 & $1.8: 1: 1.2$ \\
P2 & $10: 1: 2$ & 14600 & 2.41 & $1.7: 1: 2.3$ \\
P3 & $9: 1: 3$ & 11100 & 2.27 & $1.5: 1: 2.8$ \\
P4 & $5: 2: 1$ & 24000 & 1.58 & $1.2: 1.9: 1$
\end{tabular}

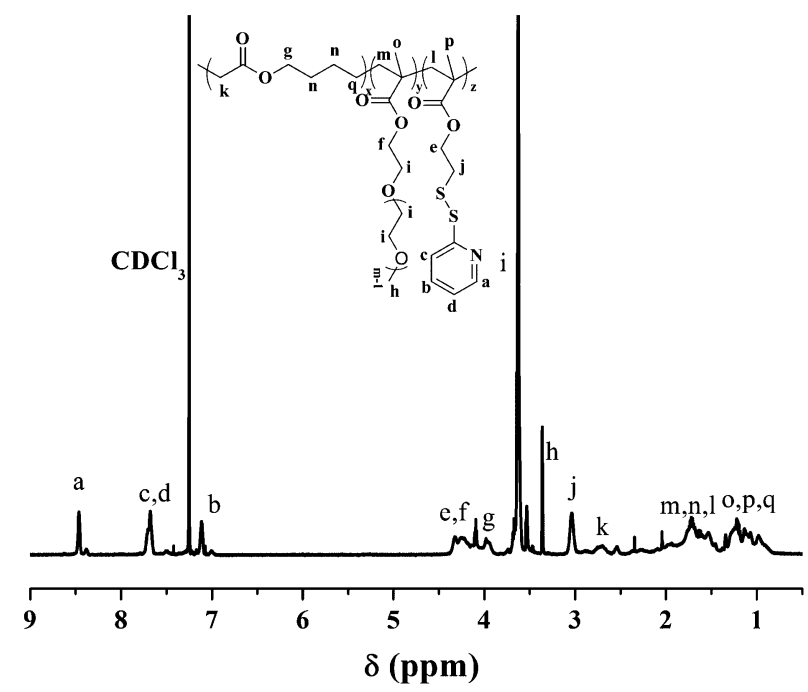

Fig. $1{ }^{1} \mathrm{H}$ NMR spectrum of the terpolymer P(MDO-co-PEGMA-COPDSMA).

compared to the methacryl radical. The MDO radical is destabilized as compared to the methacryl radical due to the presence of two electron-rich oxygen atoms as its neighbours. Sometimes MDO is able to polymerize ring-retaining as well as ring-opening. However, no peak between 100-110 ppm was seen in ${ }^{13} \mathrm{C}$ NMR spectra of P3 in this research, thereby showing the complete ring-opening reaction to form ester linkages (Fig. S12†). ${ }^{31}$

Finally, DOX-conjugated P(MDO-co-PEGMA-co-PDSMA) was obtained by grafting Mal-DOX to P(MDO-co-PEGMA-co-PDSMA) via thiol-ene click chemistry in the presence of TCEP. The terpolymer P3 was used for DOX conjugation since there was the highest mole percent of PDSMA units in the terpolymer. The ${ }^{1} \mathrm{H}$ NMR spectrum of DOX-conjugated P(MDO-co-PEGMA-coPDSMA) (P3-DOX) is shown in Fig. 2, in which the characteristic peaks of DOX appeared after the reaction. The biodegradable polymeric prodrug was successfully synthesized. Furthermore, we can still find the characteristic peaks of pyridyl group in Fig. 2, which showed that not all the pyridyldisulfide groups were converted to thiol groups. The DOX content of the polymeric prodrug was about $3.3 \%$, as determined by fluorescence spectroscopy.

\section{In vitro enzymatic degradation of P(MDO-co-PEGMA-co- PDSMA)}

The importance of biodegradable polymers has increased markedly in biomedical fields since it is not necessary to

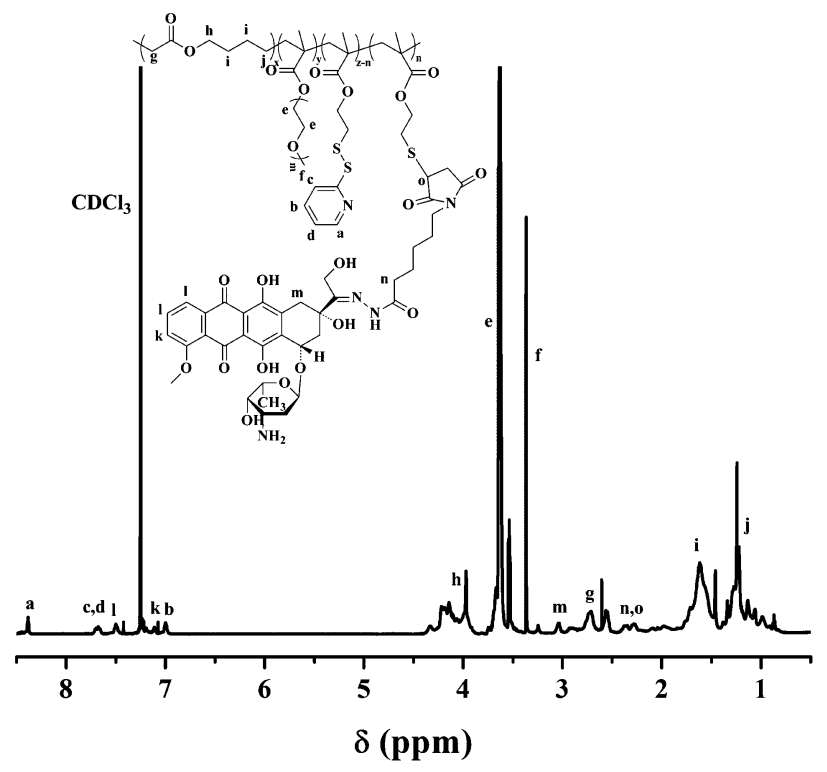

Fig. $2{ }^{1} \mathrm{H}$ NMR spectrum of the DOX-conjugated P(MDO-CO-PEGMACO-PDSMA) (P3-DOX).

remove the polymers from the body after their roles have been achieved. The enzymatic degradation of the terpolymer P4 was further investigated. The molecular weights of the terpolymers were measured before and after degradation. After incubation at $37^{\circ} \mathrm{C}$ in the presence of lipase for 3 days, the polymers left after hydrolysis showed the GPC curves with the peaks shifted to significantly lower molecular weights (Fig. 3), which showed the successful enzymatic degradation of the MDO-based polyester P(MDO-co-PEGMAco-PDSMA).

\section{Self-assembly of the polymeric prodrug micelles}

Because of the amphiphilic property of the DOX-conjugated $\mathrm{P}(\mathrm{MDO}$-co-PEGMA-co-PDSMA), the self-assembly behavior of the polymeric prodrug (P3-DOX) was investigated. The micellar aggregates were formed and measured by DLS and TEM. As shown in Fig. 4, DLS results indicated that the intensity-average hydrodynamic diameter $\left(D_{\mathrm{h}}\right)$ of the prodrug micelles was $180 \mathrm{~nm}$ with a polydispersity index (PDI) of 0.23 , which was suitable for drug delivery via the EPR effect. Moreover, the TEM images of the prodrug micelles revealed the presence of presumably spherical morphologies (Fig. 5). The mean number-average diameter was $150 \pm 30 \mathrm{~nm}$, which was much smaller than the $D_{\mathrm{h}}$ measured by DLS. The reason of the difference might be attributed to the 


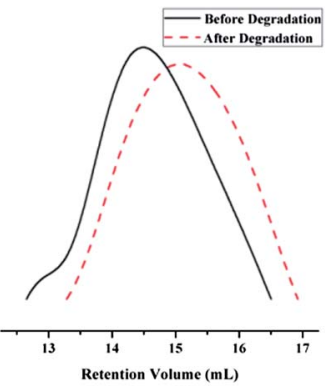

Fig. 3 GPC traces of the terpolymer of P4 before and after enzymatic degradation.

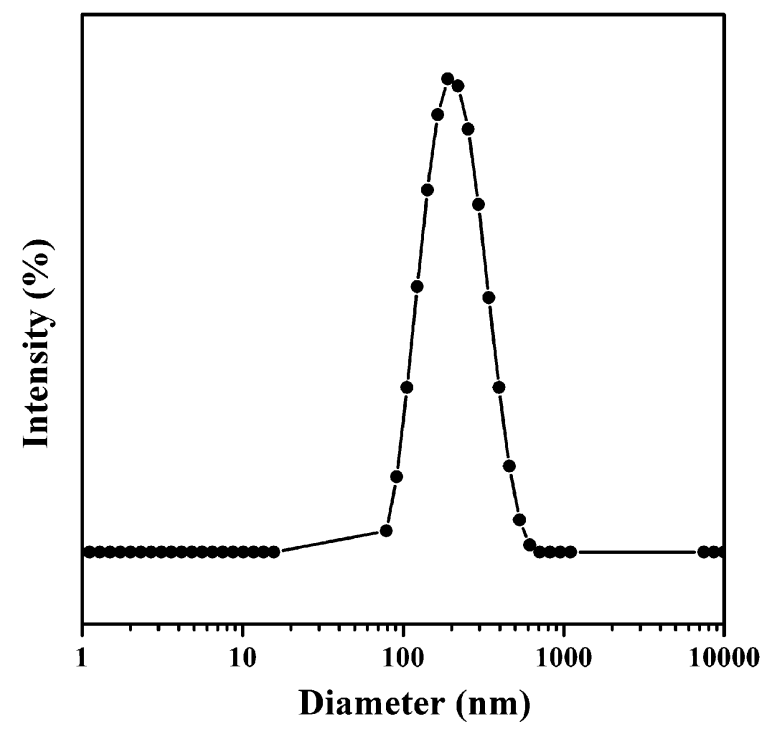

Fig. 4 DLS plots of the DOX-conjugated prodrug micelles.

different measurement status. DLS was measured in aqueous solution, while TEM was measured under a dry status, which might cause shrinkage of the hydrophilic poly(PEGMA) shell.

\section{In vitro $\mathrm{pH}$-sensitive drug release and cellular uptake}

Whether the drug can be released from the drug nanocarriers effectively is an important parameter for the drug delivery systems. In this research, DOX was conjugated to the terpolymer via $\mathrm{pH}$-sensitive hydrazone bonds, which could be cleaved at lysomal $\mathrm{pH}$ environment. It was expected that the prodrug micelles were sufficiently stable at physiological $\mathrm{pH}$ (pH 7.4), whereas they were able to rapidly release DOX in response to endosomal $\mathrm{pH}(\mathrm{pH}$ 5.5). Therefore, the release of DOX from the polymeric prodrug micelles P3-DOX was studied under different conditions, i.e., $\mathrm{pH} 5.5$ and $\mathrm{pH} 7.4$ at $37{ }^{\circ} \mathrm{C}$. The results showed that the release of DOX in a mildly acidic environment was significantly faster than that at neutral pH (Fig. 6). For example, approximately 50\% of DOX was release in $24 \mathrm{~h}$ at pH 5.5. After $24 \mathrm{~h}$, most of the hydrazone bonds were hydrolyzed and the release of DOX became

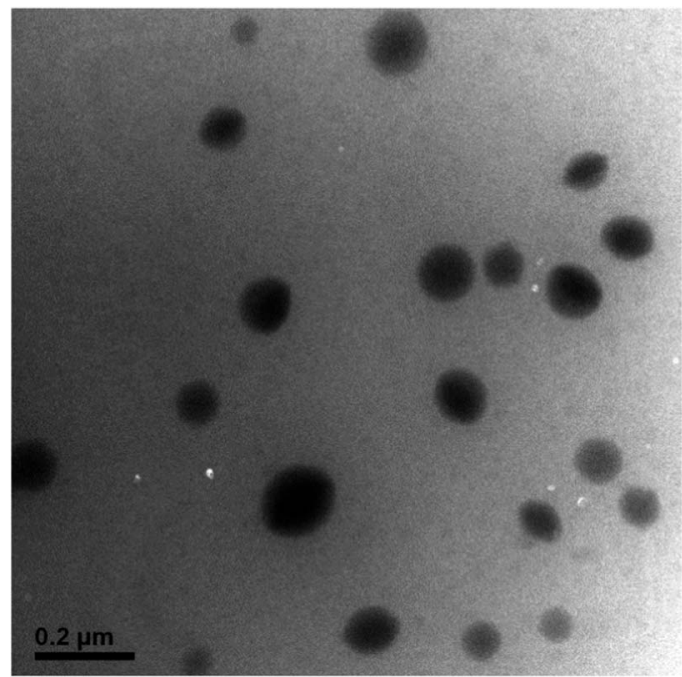

Fig. 5 TEM image of the DOX-conjugated prodrug micelles.

slower. In contrast, only $10 \%$ of DOX was released at $\mathrm{pH} 7.4$ under the same conditions. This might be attributed to the $\mathrm{pH}$-sensitive hydrazone bonds. These results indicated that the prodrug micelles were sufficiently stable under physiological conditions and quickly activated in lysosomal compartments.

The cellular uptake and intracellular drug release of DOX were studied by flow cytometry and fluorescence microscopy. DOX itself is fluorescent and it can be used directly to measure cellular uptake without adding additional markers. In general, the fluorescent intensity is proportional to the amount of DOX that internalized by the cells. High DOX concentration $\left(10 \mu \mathrm{g} \mathrm{mL}^{-1}\right)$ was chosen for the cellular uptake experiments in order to obtain strong fluorescence of DOX in the cells. Flow cytometric results of cell-associated

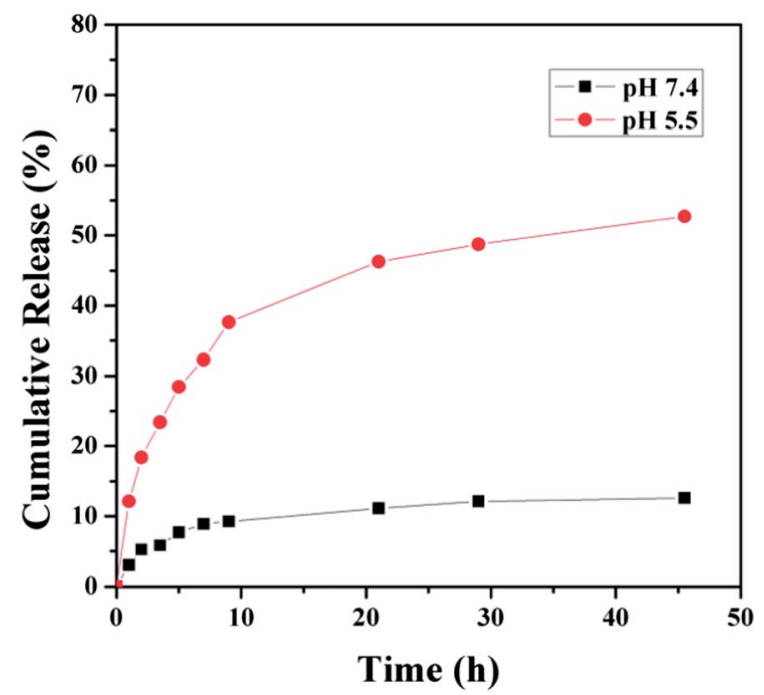

Fig. 6 In vitro cumulative release of DOX from DOX-conjugated prodrug micelles under different $\mathrm{pH}$ conditions ( $\mathrm{pH} 7.4$ and 5.5). 
DOX fluorescence for A549 cells are shown in Fig. 7. The relative geometrical mean fluorescent intensities of cells pretreated with the prodrug micelles increased when the incubation time was prolonged. Furthermore, the internalization of the prodrug micelles was evaluated by fluorescence microscopy. A549 cells were incubated with the same concentration of the prodrug for $2 \mathrm{~h}, 4 \mathrm{~h}$ and $6 \mathrm{~h}$. As shown in Fig. 8, red fluorescence of DOX can be observed in the cells after $2 \mathrm{~h}$ of incubation, which demonstrated that the prodrug micelles were successfully internalized by A549 cells. After being incubated for $6 \mathrm{~h}$, stronger fluorescence of DOX was observed in the cells. More importantly, DOX accumulated in

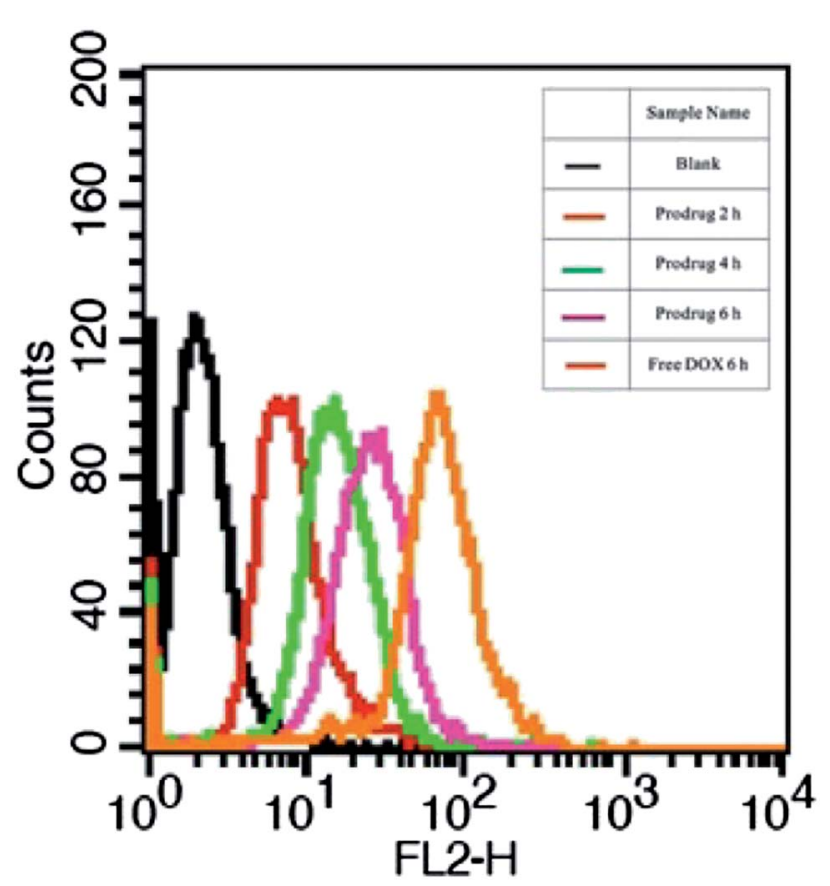

Fig. 7 Flow cytometric histogram profiles of A549 cells incubated with polymeric prodrug micelles and free DOX for different time intervals (DOX concentration $=10 \mu \mathrm{g} \mathrm{mL}^{-1}$ ).
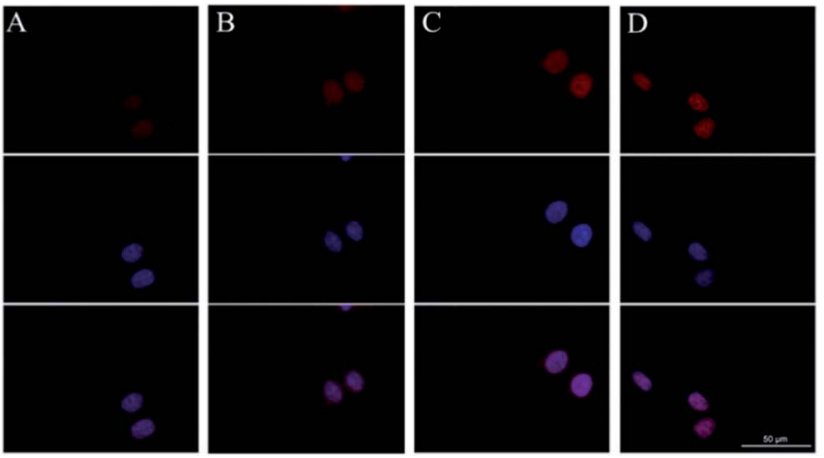

Fig. 8 Fluorescence microscopy images of A549 cells incubated with the polymeric prodrug micelles and free DOX $\left(10 \mu \mathrm{g} \mathrm{mL}^{-1}\right)$. From top to bottom: DOX (red), DAPI (blue) and a merge of the two images. (A) Prodrug, 2 h; (B) prodrug, 4 h; (C) prodrug, 6 h; and (D) free DOX, $6 \mathrm{~h}$. the nucleus and exhibited the same effect as free DOX, which suggested that DOX can be cleaved from the prodrug and then escape from the lysosome. DOX can bind with DNA and then inhibit the replication of DNA. Therefore, the rapid lysosomal escape of DOX is very important to inhibit cancer cell proliferation effectively. Both flow cytometry and fluorescence microscopy confirmed that the polymeric prodrug micelles can be efficiently internalized by A549 cells.

\section{Cell viability assays}

The excellent biocompatibility of polymeric micelles is very important for drug delivery applications. The cytotoxicity of the polymer precursor P(MDO-co-PEGMA-co-PDSMA) was evaluated by MTT assay after incubation of HUVECs with $\mathrm{P}$ (MDO-co-PEGMA-co-PDSMA) micellar solution for $48 \mathrm{~h}$. As shown in Fig. 9, the terpolymer P(MDO-co-PEGMA-coPDSMA) was nontoxic to HUVECs even at high polymer concentration (1 $\left.\mathrm{mg} \mathrm{mL} \mathrm{mL}^{-1}\right)$, indicating that the polymer precursor showed excellent biocompatibility and low cytotoxicity.

Furthermore, the DOX-conjugated P(MDO-co-PEGMA-coPDSMA) P3-DOX, which can self-assemble to prodrug micelles, was further investigated to evaluate the potential therapeutic efficacy. In vitro cytotoxicity of the polymeric prodrug micelles compared with that of free DOX was determined by MTT viability assay against A549 cancer cells. The DOX-conjugated prodrug micelles can strongly inhibit the proliferation of A549 cells. They showed similar therapeutic efficacy as free DOX, with approximately $80 \%$ cell death, when the DOX concentration was as low as $5 \mu \mathrm{g} \mathrm{mL}$ (Fig. 10). The results above showed that DOX released from the polymeric prodrug micelles could exhibit as potent drug efficacy as free DOX after internalization into the A549 cancer cells, producing the desired pharmacological action and avoiding the side effects of free DOX.

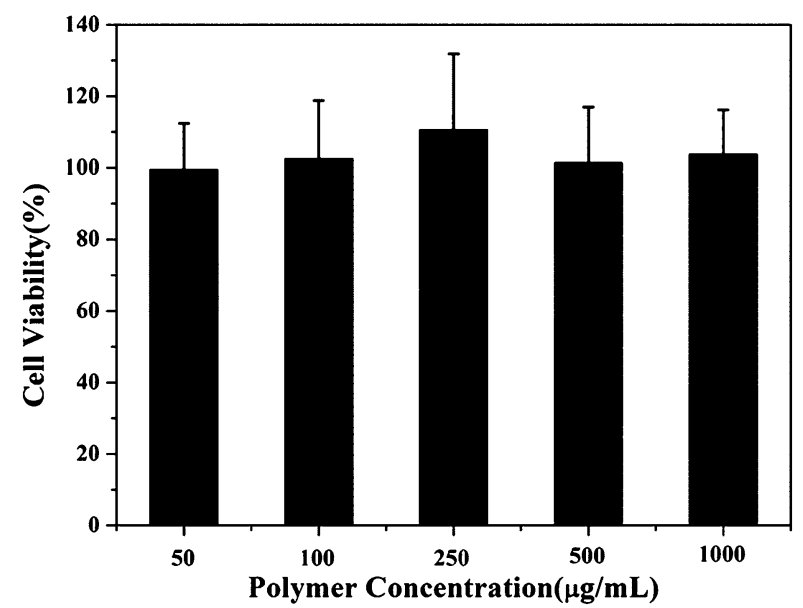

Fig. 9 Cytotoxicity of P(MDO-CO-PEGMA-CO-PDSMA) against HUVECs after $48 \mathrm{~h}$ incubation at various concentrations. 


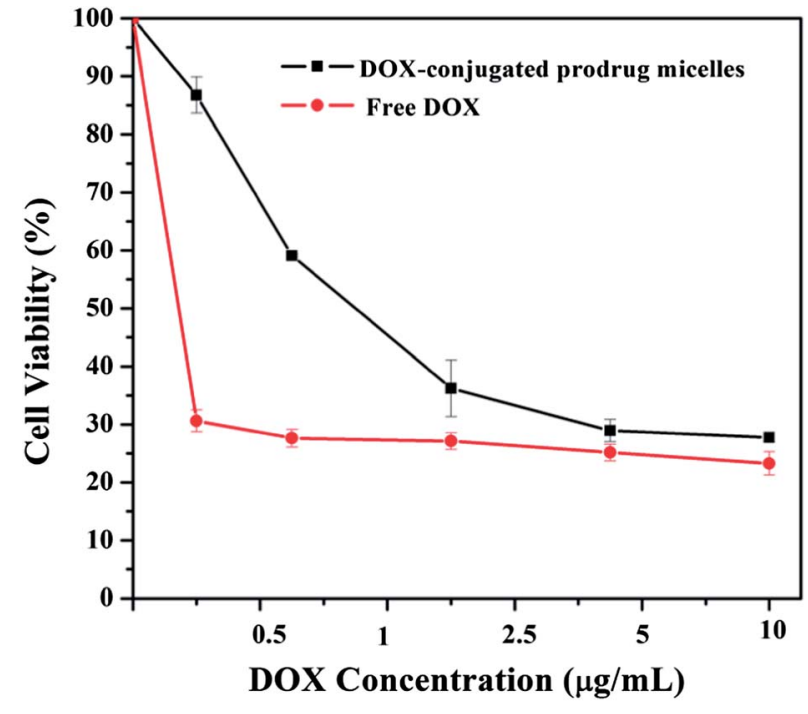

Fig. 10 The cell viability of the polymeric prodrug micelles and free DOX against A549 cancer cells after $48 \mathrm{~h}$ incubation at various concentrations.

\section{Conclusions}

In conclusion, biodegradable, biocompatible, functional polyesters P(MDO-co-PEGMA-co-PDSMA) were successfully synthesized via radical ring-opening polymerization of MDO, PEGMA and PDSMA. This new kind of functional polyester can be degraded in the presence of lipase. DOX-conjugated $\mathrm{P}\left(\mathrm{MDO}-\mathrm{co}^{-}\right.$ PEGMA-co-PDSMA) (P3-DOX) was successfully synthesized by conjugating Mal-DOX to $\mathrm{P}$ (MDO-co-PEGMA-co-PDSMA) via thiol-ene click chemistry. The DOX conjugated $\mathrm{P}\left(\mathrm{MDO}-\mathrm{co}^{-}\right.$ PEGMA-co-PDSMA) P3-DOX can self-assemble into prodrug micelles with the $D_{\mathrm{h}}$ of $180 \mathrm{~nm}$. The in vitro drug release showed that the release of DOX was much faster at lysosomal $\mathrm{pH}$ than that at normal physiological environment because of the cleavage of hydrazone bonds. The polymeric prodrug micelles can be easily internalized by A549 cells as demonstrated by flow cytometry and fluorescence microscopy. Furthermore, MTT results showed that the DOX conjugated prodrug micelles can strongly inhibit the proliferation of A549 cells and had similar drug efficacy as free DOX. Because of the simple one-pot preparation of the functional MDO-based polyesters with excellent biocompatibility and biodegradability, this new kind of functional poly(vinyl-co-ester)s shows great potential in biomedical fields, such as drug delivery, tissue engineering, and gene therapy.

\section{Acknowledgements}

Financial support from the National Natural Science Foundation of China (nos 51333005, 21174126, 51103126, 21374095, 51303154, and 51025312), the National Basic Research Program of China (2011CB606203), the Research Fund for the Doctoral Program of Higher Education of China (20110101110037, 20120101130013, and 20130101120177) and the Fundamental
Research Funds for the Central Universities (no. 2013QNA4047) are gratefully acknowledged.

\section{Notes and references}

1 M. C. Serrano, E. J. Chung and G. A. Ameer, Adv. Funct. Mater., 2010, 20, 192-208.

2 H. Y. Tian, Z. H. Tang, X. L. Zhuang, X. S. Chen and X. B. Jing, Prog. Polym. Sci., 2012, 37, 237-280.

3 B. L. Guo, L. Glavas and A. C. Albertsson, Prog. Polym. Sci., 2013, 38, 1263-1286.

4 C. M. Dong and G. Liu, Polym. Chem., 2013, 4, 46-52.

5 J. K. Oh, Soft Matter, 2011, 7, 5096-5108.

6 S. Agarwal, Polym. Chem., 2010, 1, 953-964.

7 J. Y. Huang, R. Gil and K. Matyjaszewski, Polymer, 2005, 46, 11698-11706.

8 J. F. Lutz, J. Andrieu, S. Üzgün, C. Rudolph and S. Agarwal, Macromolecules, 2007, 40, 8540-8543.

9 H. Wickel, S. Agarwal and A. Greiner, Macromolecules, 2003, 36, 2397-2403.

10 S. Jin and K. E. Gonsalves, Macromolecules, 1997, 30, 31043106.

11 L. Q. Ren, S. Speyerer and S. Agarwal, Macromolecules, 2007, 40, 7834-7841.

12 V. Delplace, A. Tardy, S. Harrisson, S. Mura, D. Gigmes, Y. Guillaneuf and J. Nicolas, Biomacromolecules, 2013, 14, 3769-3779.

13 C. Riachi, N. Schüuwer and H. A. Klok, Macromolecules, 2009, 42, 8076-8081.

14 S. Borkar, A. Sen and J. R. Shallenberger, J. Polym. Sci. Part A: Polym. Chem., 2006, 44, 1225-1232.

15 Q. Jin, S. Maji and S. Agarwal, Polym. Chem., 2010, 3, 27852793.

$16 \mathrm{~J}$. Undin, A. Finne-Wistrand and A. C. Albertsson, Biomacromolecules, 2013, 14, 2095-2102.

17 X. Pang, H. L. Du, H. Q. Zhang, Y. J. Zhai and G. X. Zhai, Drug Discov. Today, 2013, 18, 1316-1322.

18 Y. D. Gu, Y. N. Zhong, F. H. Meng, R. Cheng, C. Deng and Z. Y. Zhong, Biomacromolecules, 2013, 14, 2772-2780.

19 J. Rautio, H. Kumpulainen, T. Heimbach, R. Oliyai, D. Oh, T. Järvinen and J. Savolainen, Nat. Rev. Drug Discov., 2008, 7, 255-270.

20 J. Y. Fang and S. A. Al-Suwayeh, Expert Opin. Drug Del., 2012, 9, 657-669.

21 V. Delplace, P. Couvreur and J. Nicolas, Polym. Chem., 2014, 5, 1529-1544.

22 Y. Bae, S. Fukushima, A. Harada and K. Kataoka, Angew. Chem. Int. Ed., 2003, 42, 4640-4643.

23 X. L. Hu, S. Liu, Y. B. Huang, X. S. Chen and X. B. Jing, Biomacromolecules, 2010, 11, 2094-2102.

24 T. Jiang, Y. M. Li, Y. Lv, Y. J. Cheng, F. He and R. X. Zhuo, Colloid. Surface B, 2013, 10, 542-548.

25 J. Z. Du, X. J. Du, C. Q. Mao and J. Wang, J. Am. Chem. Soc., 2011, 133, 17560-17563.

26 H. B. Wang, Y. Wang, Y. J. Chen, Q. Jin and J. Ji, Polym. Chem., 2014, 5, 854-861. 
27 W. J. Bailey, S. R. Wu and Z. D. Ni, Makromol. Chem., 1982, 183, 1913.

28 Z. F. Jia, L. J. Wong, T. P. Davis and V. Bulmus, Biomacromolecules, 2008, 9, 3106-3113.

29 D. Willner, P. A. Trail, S. A. Hofstead, H. D. King, S. J. Lasch, G. R. Braslawsky, R. S. Greenfield, T. Kaneko and R. A. Firestone, Bioconjugate Chem., 1993, 4, 521-527.

30 S. Ghosh, S. Basu and S. Thayumanavan, Macromolecules, 2006, 39, 5595-5597.

31 S. Agarwal, R. Kumar, T. Kissel and R. Reul, Polym. J., 2009, 41, 650-660. 